\title{
Templating hydrogels
}

\author{
John Texter
}

Received: 1 November 2008 /Revised: 15 December 2008/Accepted: 15 December 2008 /Published online: 20 January 2009

(C) Springer-Verlag 2009

\begin{abstract}
Templating processes for creating polymerized hydrogels are reviewed. The use of contact photonic crystals and of non-contact colloidal crystalline arrays as templates are described and applications to chemical sensing and device fabrication are illustrated. Emulsion templating is illustrated in the formation of microporous membranes, and templating on reverse emulsions and double emulsions is described. Templating in solutions of macromolecules and micelles is discussed and then various applications of hydrogel templating on surfactant liquid crystalline mesophases are illustrated, including a nanoscale analogue of colloidal crystalline array templating, except that the bead array in this case is a cubic array of nonionic micelles. The use of particles as templates in making coreshell and hollow microgel beads is described, as is the use of membrane pores as another illustration of confinement templating.
\end{abstract}

Keywords Hydrogel · Templating · Photonic crystal . Emulsion templating $\cdot$ Double templating

J. Texter (ه)

Max Planck Institute for Colloids and Interfaces,

Am Muehlenberg 1,

14476 Golm-Potsdam, Germany

e-mail: jtexter@emich.edu

J. Texter

School of Engineering Technology, Eastern Michigan University, Ypsilanti, MI 48197, USA

\section{Introduction}

The syntheses of novel zeolites and nanoporous materials by templating on surfactant mesophases has been well established and very extensively reviewed [1-4]. The dynamic range in pore size is controlled by the length scale of the surfactants used in the templating, and typically are limited to lengths less than $100 \mathrm{~nm}$ and more usually to less than $30 \mathrm{~nm}$.

Advances in pore size control were obtained when it was found that photonic crystals derived from bead arrays [5] and random bead arrangements [6] could be used to template diverse nanoporous, mesoporous, and microporous materials. Here the bead diameter controls the length scale of the final pores obtained.

In both of these approaches, the underlying templates provide a negative image of the final pore structure and the interstices constitute the volumes that are filled with a new phase during the initial stages of the templating chemistry. Almost any conceivable phase may be inserted in mesoporous to microporous bead assemblies [7,8]. Once the interstices are filled with a new phase, the templating phase, surfactant mesophase, or bead assembly, are removed by dissolution or by pyrolysis. If the templating phase is a metal oxide, it is usually dissolved by aqueous $\mathrm{HCl}$ or other acid (aqueous HF in the cases of silica and titania); if the templating phase is a polymer that dissolves (such as a polystyrene (PS) in toluene) the composite is subjected to dissolution by being placed in a solvent bath. If the templated phase is inorganic and thermally robust, a polymeric templating phase is removed by pyrolysis. The filling of mesophase interstices by organic polymerization has recently been reviewed [9-10].

In this review we focus upon templating involving polymeric organic hydrogels. Hydrogels are important 
classes of composite materials for many reasons. Foremost among these is the utility hydrogel coatings provide in facilitating interfacing synthetic materials with biological tissues [11]. No less importantly is the role hydrogels play in drug delivery systems, particularly in retarding the adsorption of proteins onto injected and implanted surfaces. Hydrogels also offer diverse applications in device fabrication and in chemical and physical testing.

\section{Templating on photonic crystals}

We consider two general types of photonic crystals fabricated by bead assemblies. The first is the type wherein the beads are in physical contact with their nearest neighbors (12 nearest neighbors in the fcc or in the hcc structures). This type of structure offers the greatest number of degrees of freedom in producing new materials. We denote such systems as "contact" photonic crystals, since nearest neighbors are in physical contact with each other. The second type of photonic material we examine is derived from crystalline arrays of charged beads, where nearest neighbors do not physically contact one another. These materials are known as colloidal crystalline arrays, CCA. In this type of material the beads also adopt an fcc or bcc lattice structure. Such arrays of charged beads form under stringent conditions of low ionic strength in aqueous suspension or in other solvents. Charge repulsion between such beads induces crystallization.

One of the chief limiting factors in utilizing photonic crystals of the first type is the tremendous activation present in their crystallization or 3D assembly. Debord and Lyon [12] used the thermal responsiveness of hydrogel particles in an attempt to circumvent some of this activation. Centrifugation was used to physically concentrate the particles, and then this concentrate was subject to a number of heating and cooling cycles. The PNIPAM poly ( $N$ isopropyl acrylamide) hydrogel is known to condense or compress upon heating and it expands upon cooling. These cycles provided thermally induced mechanical annealing and greatly increased the crystallinity of the concentrated assembly.

$3 D$ contact photonic crystal templating Takeoka and Watanabe [13] used silica (contact) photonic crystals to template crosslinked hydrogels. $N$-isopropylacrylamide (NIPAM) and methylene bisacrylamide (MBA) were dissolved in 1,4-dioxane and perfused through air-dried silica photonic crystals and then polymerized using benzoyl peroxide as thermal initiator. After polymerization was completed, these silica-hydrogel composites were soaked in $5 \%$ aqueous $\mathrm{HF}$ to dissolve the templating silica. The resulting hydrogel with periodic strucuture (a reverse opal structure of voids left by the silica) exhibited structural colors resulting from Bragg reflections in the visible. Due to the changes in refraction index at hydrogel/void boundaries, these gels mostly were opaque to transmitted light, but iridescent to reflected light because of this periodic (photonic crystal) structure. This iridescence is illustrated in Fig. 1. Silica particles of $0.5,4,6$, and $10 \mu \mathrm{m}$ diameter were used in preparing templating photonic crystals and subsequently templated hydrogels. Optical micrographs proved the periodic structures of the final hydrogels [13]. The porous structure greatly facilitated volume equilibration of these templated gels, in comparison to bulk gel samples, when subjected to changes in temperature. The authors observed facilitated equilibration to the volume changes occurring at a rate 1,000-fold faster for the templated gels. The periodic porous structure was retained on thermal cycling through the volume phase transition domain.

Contact photonic crystals built from 450-nm diameter polystyrene beads were grown in a flow cell [14]. The resulting crystals were used to template polyacrylamide by infusing aqueous $47.9 \%$ acrylamide, $3.8 \%$ methylene bisacrylamide, and $0.4 \%$ diethoxyacetophenone (photoinitiator) solution into the interstices. UV irradiation was used to drive polymerization. The templating beads were removed by soaking in toluene. The resulting inverse opal hydrogel is soft but has a sufficiently strong modulus that it can be handled. Its reflectance spectrum in the visible shifts with humidity, as the hydrogel equilibrates at different water uptakes and swelling states as humidity varies. This photonic material can thus be used as a relative humidity

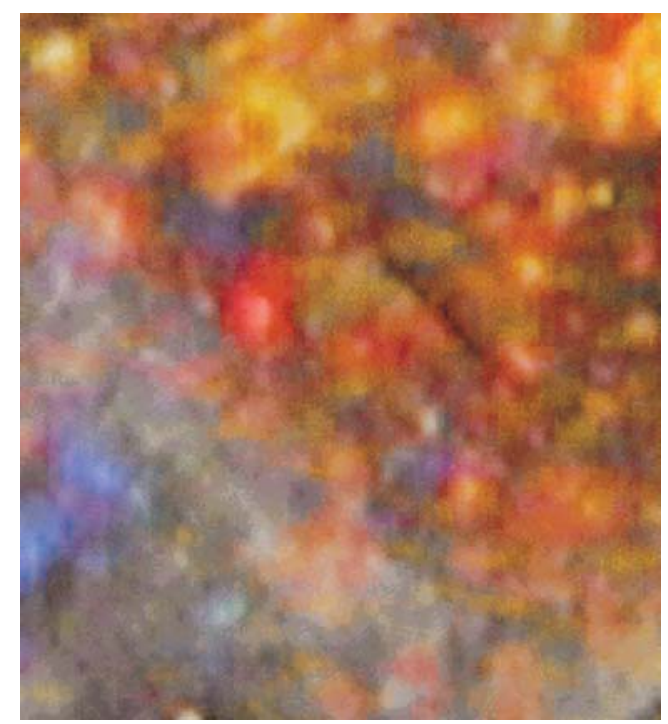

Fig. 1 Iridescent color resulting from the diffraction and scattering of visible light by the periodic structure of the PNIPAM/bisacrylamide crosslinked gel. Reproduced by permission from [14]; copyright 2002 by the American Chemical Society 
sensor. Similar hydrogel sensors templated on contact photonic crystals were used by Braun and coworkers to fabricate colorimetric $\mathrm{pH}$ [15] and glucose [16] sensors. Recently Matsubara et al. fabricated temperature sensitive hydrogels using similar inverse opal templating methods for crosslinked PNIPAM hydrogels that also incorporated monomers bearing azobenzene groups, making the hdyrogels photoresponsive [17].

$2 D$ contact photonic crystal templating A very interesting double templating method for preparing 2D arrays of microgel beads was demonstrated by Sakai et al. [18]. A hexagonally close-packed array of silica beads (the first template) was produced by simple drying of an aqueous suspension of monodisperse silica beads. After drying, this monolayer was infused with styrene so that the styrene film just covered the bead monolayer. This styrene was then polymerized using thermal initiation over $30 \mathrm{~h}$ at $40{ }^{\circ} \mathrm{C}$. This silica-polystyrene film was then soaked in 5\% aqueous $\mathrm{HF}$ to dissolve the silica, and to produce the second template, the polystyrene encased negative image of the silica bead hexagonal array. A glass slide is then activated by coating with a silane-propylmethacrylate layer. The airdried polystyrene template is then filled with NIPAM and MBA in ethanol, containing a photoinitiator (2,2-dimethoxy-1,2-diphenylethan-1-one; Irgacure 651), sandwiched to the activated glass slide, and polymerized under a UV lamp. The polystyrene template was then removed by dissolving it in toluene and the crosslinked hydrogel was soaked in water. Because of the glass slide activation, the monolayer of hexagonally arranged microgel beads is chemically attached to the slide. When the beads are subjected to temperature cycling they swell to very tightly pack at, for example, $20^{\circ} \mathrm{C}$, and shrink to separated beads at high temperature $\left(40^{\circ} \mathrm{C}\right)$ (Fig. 2).

It is noteworthy that Tsuji and Kawaguchi [19] developed an alternative method to produce monolayers of microgel bead arrays. In their work the separation distance between beads was controlled by the length/thickness of a surface layer of hairy polymer.

$3 D$ templating on colloidal crystalline arrays Asher and coworkers in an extensive body of work have shown that fcc colloidal crystalline arrays of submicron to multimicron diameter beads may be imparted a solid modulus by polymerizing hydrogels in the aqueous spaces between the beads [20]. While such CCA have been known for some time [21, 22], it was the templating of hydrogels provided by Asher and coworkers that have made such CCA much more useful, since they now can be handled as soft solid materials.

Because of the suspension and fluidic nature of CCA, thermally initiated polymerization of hydrogels in the aqueous fluid phase generally is too disruptive to the crystalline array and leads to unsatisfactory degradation of crystalline order among the beads. For this reason UV irradiation has usually been used to effect hydrogel polymerization. This need has been partially overcome by using PS particles that contain superparamagnetic iron oxide nanoparticles and a locally applied magnetic field gradient [23]. The charged PS/iron oxide composite particles self assemble into CCA in deionized water, but the packing is significantly affected by proximal magnetic field gradients. A locally applied uniform magnetic field was found to induce interparticle magnetic moments that produced a repulsive force in the plane perpendicular to the magnetic field and an attractive force in the direction parallel to the field [24]. This innovative use of applied fields makes it possible to obtain CCA self-assembly under high ionic strength conditions, when crystallization would otherwise not be obtained due to insufficient repulsion between beads. Foulger and coworkers introduced the use of PEG-based hydrogel monomers as alternatives to acrylamide-based hydrogels in such CCA-based templating
Fig. 2 Fabrication process of a 2D monolayer of gel beads by the double-template polymerization method. The optical micrograph illustrates the product gel beads. Reproduced by permission from [19]; copyright 2007 by the American Chemical Society
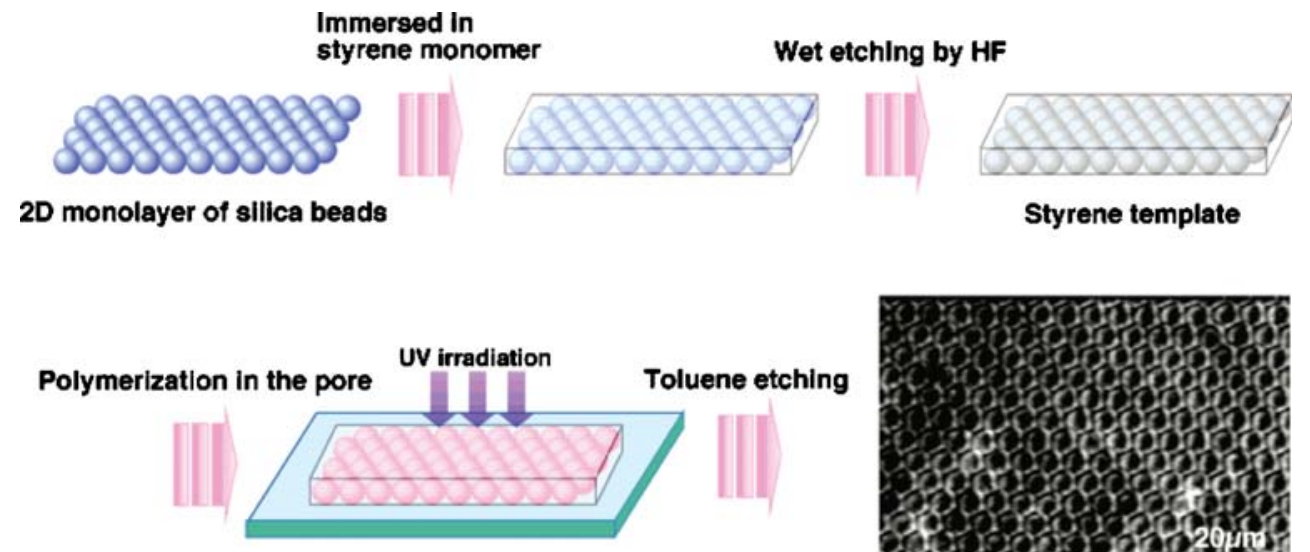

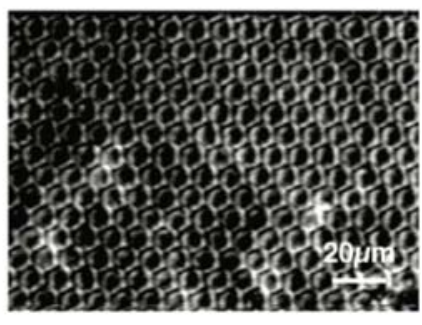

2D monolayer of gel beads 
[25]. Such a PEG-monomer-templated hydrogel was used to demonstrate mechanicolorimetric responses of the PCCA (polymerized CCA) [26]. Foulger and coworkers also have recently reported general schemes for using Click-Chemistry to functionalize bead particles in PCCA post-hydrogel templating to incorporate large numbers of dyes per bead [27].

The use of thermoreversible hydrogels with such CCA can produce a variety of devices. Thermoreversible-based CCA in a bcc structure composed of $100 \mathrm{~nm}$ particles [28] of poly( $N$-isoproplyacrylamide), PNIPAM, provide a very temperature sensitive probe, where the narrow bandwidth extinction at about $530 \mathrm{~nm}$ increases five-fold in magnitude over the 24 to $35^{\circ} \mathrm{C}$ interval. Here the index of refraction changes dramatically as the diffuse hydrogel condenses on itself as the temperature is raised. In an alternative approach to realizing a photonic thermometer [29], PS beads of $99 \mathrm{~nm}$ diameter were used to form a CCA in a bcc lattice and a thermally reversible hydrogel, PNIPAM, was polymerized in the continuous phase to lock-in the structure. In this material the overall lattice constant changes with temperature and the peak extinction wavelength shifts from $700 \mathrm{~nm}$ to $450 \mathrm{~nm}$ as temperature shifts from 10 to $35^{\circ} \mathrm{C}$. Bead-encapsulated Oil Blue $\mathrm{N}$ has been used to make such bead arrays very sensitive to heating effects and the resulting index of refraction changes have been demonstrated to produce fast optical switching $[28,30]$. Nanosecond optical switching has also been demonstrated [31].

Such hydrogel-templated PCCA have also been suitably modified to provide chemical sensors. Doping the hydrogel monomers with ligand-monomers suitable for binding some chemical anylate generally will lead to a lattice expansion or contraction upon binding, leading to a colorimetric Bragg diffraction shift in the PCCA [24, 32-34].

An interesting double templating CCA approach has been reported by Foulger and coworkers [35]. Instead of aqueous polyacrylamide, an aqueous PEGylated methacrylate $\left(M_{\mathrm{n}}=360\right)$ is used as the monomer and a PEGylated dimethacrylate $\left(M_{\mathrm{n}}=550\right)$ is used as crosslinker in preparing photopolymerized PCCA of PS beads (109 nm diameter). The resulting PCCA was air dried and then vacuum dried and then soaked in 2-methoxyethyl acrylate (MOEA) for 2 days. The swollen sample was then infused with some EGDMA and photoinitiator and photopolymerized to produce the resulting PCCA composite photonic band gap material. This material proved useful as a novel compressive stress sensor.

Similarly, templated hydrogel composites have been used to fabricate hybrid piezoelectric actuators for rejection wavelength tuning $[36,37]$ and for mechanochromic tuning of photoluminescent dyes [38]. PCCA comprising electroactively coated silica particles were used for electroactively tuning the stop band properties of PCCA [39]. A strain sensor based on such arrays by pressure tuning [40] was also demonstrated.

\section{Hydrogels on emulsion templates}

The importance of emulsions with aqueous gel phases has been recently reviewed [41]; a major application area is in food grade emulsions and aqueous proteins are prime candidates for formulating the continuous phase hydrogels.

Emulsion templating Kim and coworkers demonstrated a supercritical $\mathrm{CO}_{2}\left(\mathrm{scCO}_{2}\right)$ in water emulsion templating method to prepare microporous hydrogels [42]. A fluronated surfactant and polyvinyl alcohol (PVA) were used as dispersing agents and a mixture of acrylamide and methylene bisacrylamide (as crosslinking agent) were introduced into the aqueous solution, along with calcium derived by dissolving $\mathrm{CaO}$ and postassium persulfate as initiator. Supercritical $\mathrm{CO}_{2}$ was introduced and emulsified and then the emulsion was heated to $60{ }^{\circ} \mathrm{C}$ and 280 bar while the aqueous phase underwent chain radical polymerization. The calcium ions formed particles of calcium carbonate that further stabilized the aqueous $/ \mathrm{scCO}_{2}$ interfaces, aiding the surfactant and PVA cosurfactant stabilization. After depressurization the $\mathrm{CO}_{2}$ escapes the matrix and leaves an open cell structure, such as that illustrated in Fig. 3. The interfacial calcium carbonate can be removed by washing the matrix with aqueous acid.

Reverse emulsion templating The recent surge in developing Pickering emulsions where nanoparticles of various

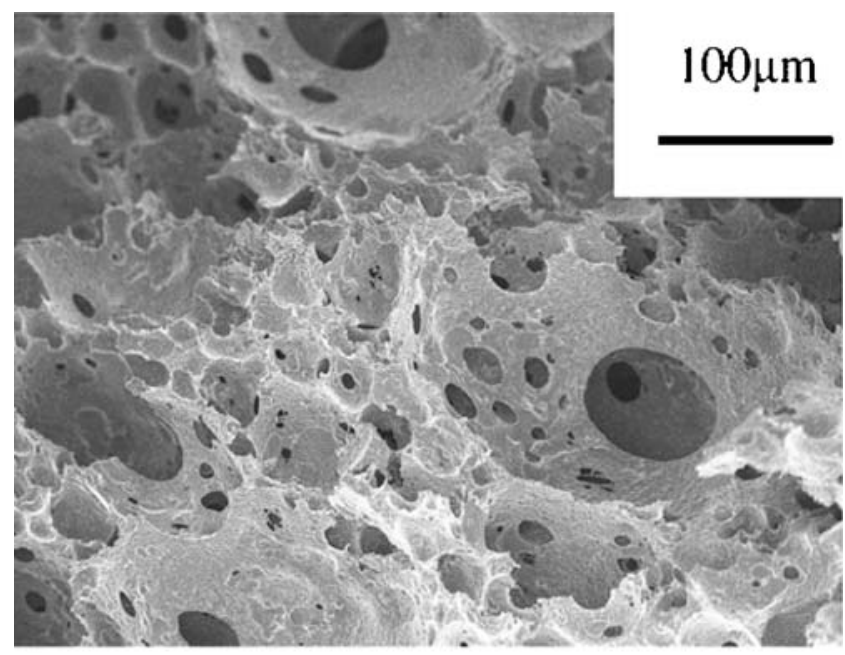

Fig. 3 SEM image of fracture surface of the $\mathrm{CO}_{2}$ templated porous $\mathrm{CaCO}_{3} / \mathrm{PAM}$ composites produced from $\mathrm{CO}_{2}$ in water emulsion with the loading of $\mathrm{Ca}(\mathrm{OH})_{2}$ was $3 \%(w / v$ to water) and the content of surfactant FC4430 was $12 \%$ ( $w / v$ to water). Reproduced by permission from [43]; copyright 2007 by Elsevier 
types are used to stabilized emulsion droplets instead of surfactants or polymeric dispersing aids has also been applied to formulating and stabilizing reverse emulsions (water in oil emulsions). Recently, hydrogel beads have been produced with shells of partially fused colloid particles, and such beads have been called colloidosomes. Applications have included aqueous agarose stabilized in shells of epoxy resin microrods [43] and agarose stabilized by polystyrene latex-based shells [44]. Further applications have included the formulation of magnetic colloidosomes using shells stabilized with nanoparticles or $\mathrm{Fe}_{3} \mathrm{O}_{4}$ [45]. It is anticipated that such colloidosomes have high potential for controlled release and drug delivery applications in addition to food and cosmetic applications [46].

Water in oil emulsion templating of hydrogel core shell beads [47] was demonstrated using alginate as the hydrogel core material and porous calcium carbonate as the shell material. Sunflower oil was used as the continuous oil phase. The colloidosome-templating process is illustrated in Fig. 4. Calcium carbonate is particularly useful as a sacrificial stabilizer, because it can easily be removed [47] by dissolution after gently suspending the crosslinked hydrogel core shell particles in a mild $\mathrm{HCl}$ solution.

Double emulsion templating Zhang and coworkers [48] demonstrated a double emulsification method to produce

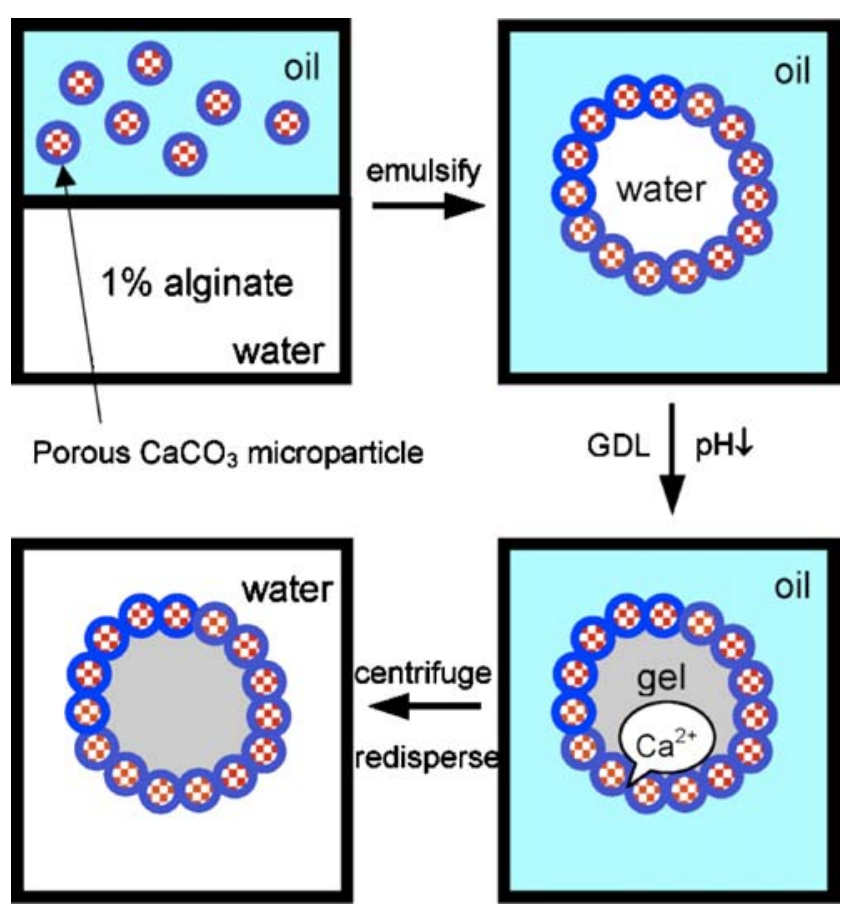

Fig. 4 Schematic illustration of the process for preparing colloidosomes based on stabilization of Pickering types of $\mathrm{CaCO}_{3}$ microparticles at the interfaces of the aqueous hydrogel droplets. Reproduced by permission from [48]; copyright 2008 by Elsevier crosslinked hydrogel porous particles. The hydrogel phase comprised sodium alginate and a small amount of hexylethylcellulose. These polymers produced an interpenetrating network that resulted in greater mechanical stability of the resulting hydrogel microparticles. An oil in water emulsion was then prepared with liquid paraffin, with oil droplets approximately $2 \mu \mathrm{m}$ in diameter being obtained. These paraffin droplets templated the ultimate pores obtained. This oil in water emulsion was then emulsified in chloroform, for example, obtaining double emulsion droplets about $100 \mu \mathrm{m}$ in diameter. This double emulsion was also mixed with aqueous calcium ion and glutaraldehyde to provide crosslinking and then the highly volatile phase was removed by drying and the paraffin was extracted with aqueous ethanol.

\section{Macromolecule and micellar templating}

Various templating methods have been demonstrated to create pores in hydrogel matrices [49-51]. Macromolecules and micelles of various sizes are included in the hydrogel monomer solution to be polymerized and then the subsequent removal of these steric blocking objects after polymerization leaves pore size distributions mirroring the dimensions of these nanotemplates. Templating gels with up to $20 \%$ sodium dodecyl sulfate (SDS) affected electrophoretic separations of SDS-protein complexes [49]. A bicontinuous phase of didodecyldimethylammonium bromide was used to template a poly(methyl methacrylate) gel [50]. More recently porous hydrogels have been studied using theoretical and simulation methods in evaluating micellar pore size effects on hydrogel creation and separation performance in GPC applications to separating globular proteins [51].

\section{Templating on LC mesophases}

Various hydrogels have been templated on liquid crystalline mesophases of surfactants where the templates are very similar to those used in preparing inorganic zeolites as discussed in the Introduction to this review. Infusion of hydrogel into the liquid crystalline interstices is done similarly as metal alkoxides in the zeolite syntheses, but the hydrogel polymerization is then done via UV irradiation or by thermal initiation, rather than by solvothermal conversion. Most of this work has been pursued by Guyman and various collaborators using templates based on the nonionic surfactant Brij 56. The phase diagram of this surfactant in water is illustrated in Fig. 5 [52], where the various LC (liquid crystalline) phases are indicated. Brij 56 is a tradename for $\mathrm{C}_{15} \mathrm{E}_{10}$. 


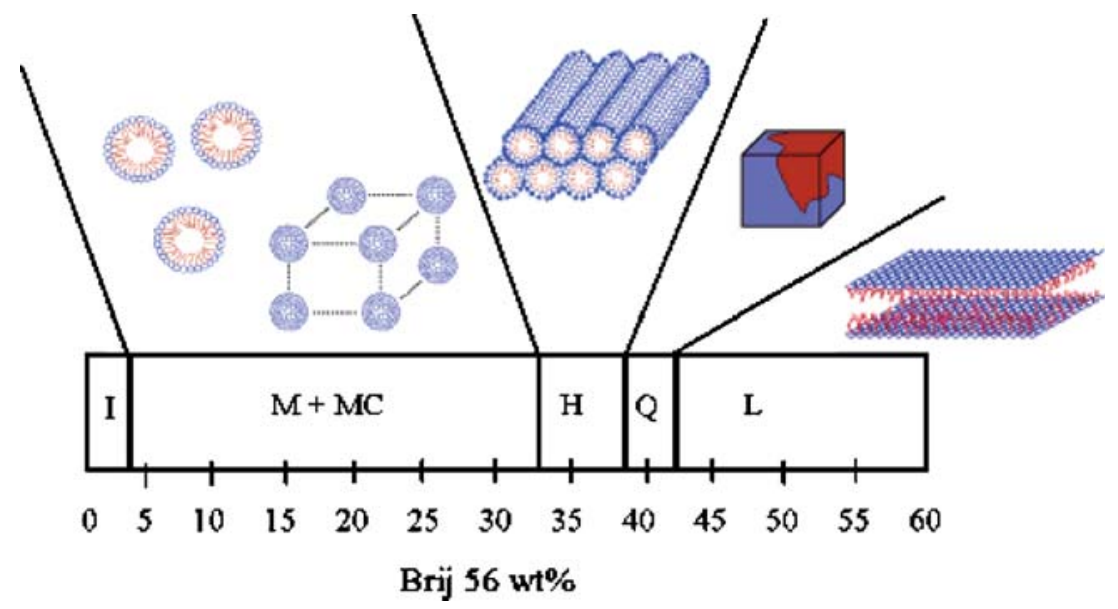

Fig. 5 Aqueous phase diagram of Brij 56 at $27{ }^{\circ} \mathrm{C}$ in $40 \%$ PEGDA. I represents an isotropic solution phase of monomeric surfactant and micelles, $M+M C$ represents a coexistence domain of micelles and cubic micellar liquid crystals, $H$ is a hexagonal phase of cylindrical

The photopolymerization of acrylamide and of hydroxyethyl methacrylate (HEMA) in various of these LC mesophases was studied in detail [53]. Both acrylamide and HEMA were found to photopolymerize more rapidly in the more highly ordered (increasing amount of Brij 56; see Fig. 5) LC mesophases. For the resulting polyacrylamide systems, the more highly ordered mesophases swelled faster and further, and they also exhibited larger mechanical storage moduli. For the polyHEMA systems, the opposite trends in swelling and mechanical moduli were observed. The porous structure obtained from the cubic phase of Brij 56 at $40 \% w / w$ surfactant is illustrated in Fig. 6 where the smooth structure obtained in solution without any added surfactant is compared with the porous structure obtained before and after surfactant removal [54]. These studies also found that faster polymerization rates resulted in retention of smaller length scales, a trend often reported in similar studies of microemulsion polymerization. micelles, $Q$ is a bicontinuous cubic phase where surfactant bilayers separate two separate continuous domains, $L$ is a lamellar phase. Reproduced by permission from [53]; copyright 2007 by the American Chemical Society

Effects of Brij 56 LC mesophase structure on templated hydrogels derived from polyethyleneglycol diacrylate (PEGDA; $M_{\mathrm{n}}=575 ; \sim 11$ oxyethylene groups between acrylates) were examined [52] using photopolymerization. Similarly to acrylamide, increases in swell rate, degree of selling, mechanical modulus, and diffusion rates were all observed to increase with increasing surfactant and increasing mesophase order.

Templating on cubic micellar gels The thermoreversible gel nature of aqueous Pluronic-type triblock copolymer systems has been very well documented since the work of Malmsten and Lindman [55]. The micelles that form selfassemble into cubic gel phases when increasing temperature induces partial dehydration of the PEO corona segments. Such segments on nearest neighbors provide interactions that produce cubic arrangements of the constituent micelles. Such thermoreversible gels were examined as possible

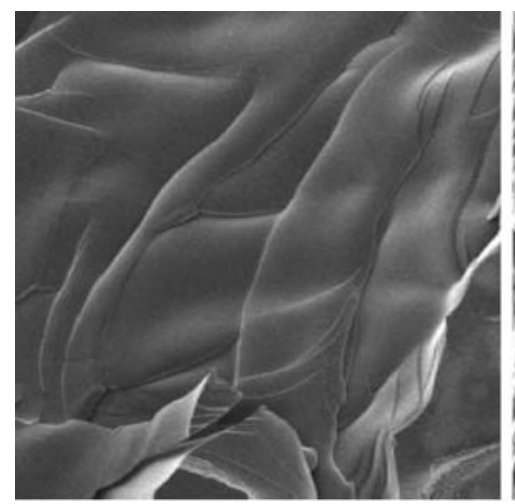

Fig. 6 SEM of photopolymerized polyacrylamide in (left) isotropic state without any Brij 56; (middle) cubic phase of Brij 56 at $40 \%$ w/w; (right) cubic phase of Brij 56 at $40 \% \mathrm{w} / \mathrm{w}$ after removal of the surfactant. Reproduced by permission from [55]; copyright 2006 by the American Chemical Society 


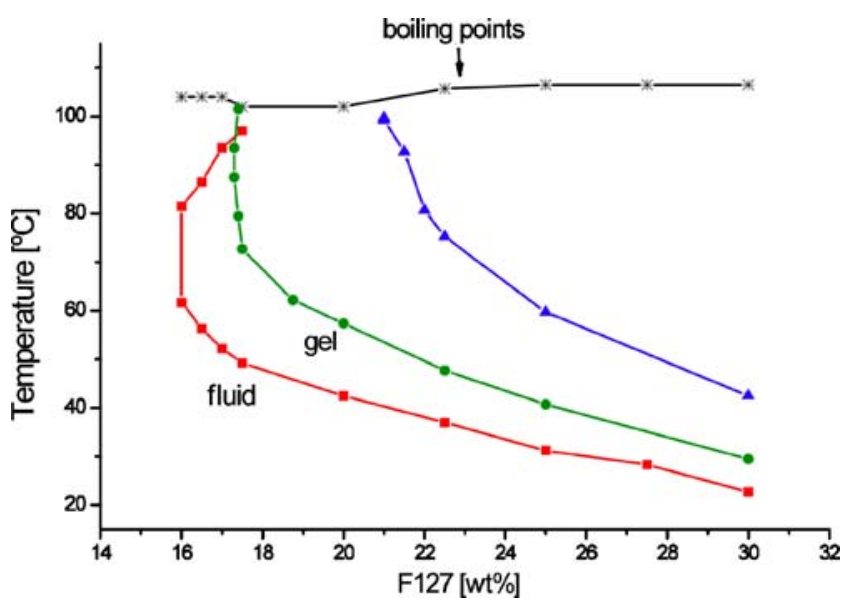

Fig. 7 Phase diagrams for F127 in 20 (filled square), 30 (filled circle), and $40 \%$ (filled triangle) $(w / w)$ aqueous acrylamide at up to $30 \%(w / w)$ F127 (Texter et al. (2006) unpublished)

fouling release coatings for marine applications, since it is known that PEO greatly retards protein adsorption, a step believed to be the beginning of biofilm formation in fouling [56]. Since such gels are thermoreversible and would quickly dissolve at lower temperatures, it was decided to see if the cubic micellar gel phase could be used to template a crosslinked hydrogel phase to better encapsulate the micelles and to thereby provide an erodible fouling release coating. Motivation for such an approach is provided by the extensive innovation of Asher et al. described above in producing practical colloidal crystalline array materials for sensors, filters, and optical switches.

Illustrated in Fig. 7 are phase diagrams for F127 in 20, 30 , and $40 \%(w / w)$ acrylamide. To the left of each of these liquid-gel boundaries is a liquid phase, with gel to the right. At $20 \%$ acrylamide, one can also pass from gel to liquid prior to boiling by increasing the temperature. At all of the concentrations where gelation occurs, it occurs thermoreversibly on increasing temperature. Basically similar phase diagrams were obtained for other hydrogel monomers, such as pentaethoxymethacrylate and hexylethoxyacrylate. In thin coatings UV polymerization was found useful. Ther- mally initiated polymerization at $90{ }^{\circ} \mathrm{C}$ was also investigated. In Fig. 8 the thermally initiated polymerization of three different compositions are illustrated. The composition in Fig. 8 (left) is almost transparent, exhibiting a bluish Tyndall effect where there has been some small amount of ripening. The composition in Fig. 8 (right) is opaque and white, and indicates the onset of microphase separation. The essential retention of transparency in the one case illustrated means that the hydrogel templating was achieved without significant disruption of its underlying cubic phase structure.

\section{Templating on particles}

Gold nanoparticles were used by Singh and Lyon [57] as templates for producing hollow PNIAM beads. Citrate stabilized gold nanoparticles are first coated with amineterminated oligomers of $\mathrm{N}$-isopropylacrylamide at room temperature where the oligomers are in an expanded random coil state. The temperature is then raised to $70{ }^{\circ} \mathrm{C}$ so that the adsorbed oligomers condense upon the surface to form a layer upon which to nucleate further PNIPAM condensation. NIPAM monomer and optional comonomers (acrylic acid, 4-acrylomido-fluoresein) and methylene bisacrylamide as crosslinker with ammonium persulfate as initiator are added and a shell of crosslinked PNIPAM is condensed around the surface modified gold nanoparticles. After separating the shelled nanoparticles by centrifugation, the $\mathrm{Au}$ cores are dissolved with aqueous KCN.

Liposomes have been used to template alginate microgel beads [58]. Liposomes are first prepared in a solution of sodium alginate. The liposomes are then isolated, washed, and suspended in a calcium solution, where the calcium ions diffuse into the liposomes and physically crosslink the alginate when the liposomes are subjected to heating and cooling cycles that cross the $T_{m}$ of the bilayer membrane. Studies using different liposomes sizes shows that one to one copies of the liposome cores were made by the alginate microgels.
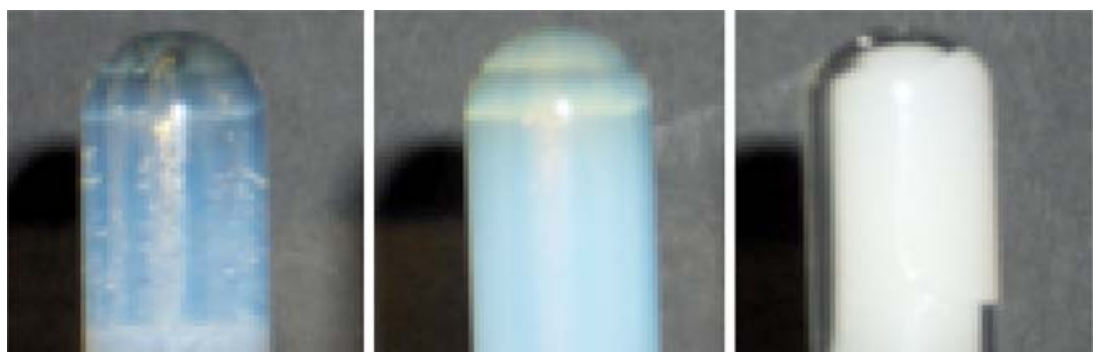

Fig. 8 Polymerization with thermal initiation at $90{ }^{\circ} \mathrm{C}$ of (left) 25\% F127, 20\% PEG5MA, 5\% PEG400DA; (middle) 20\% F127, 20\% PEG5MA, 5\% PEG400DA; (right) 20\% F127, 40\% PEG5MA, 5\% PEG400DA (Texter et al. (2006) unpublished) 


\section{Confinement templating in pores}

Using membrane pores to template by confinement has been very successfully applied to making metallic nanorods of various compositions and to making conducting polymer rods. Yan and coworkers have shown [59] that membrane pores can also template PNIPAM microgels. Polycarbonate membranes $10-\mu \mathrm{m}$ thick and having $0.8-\mu \mathrm{m}$ diameter pores were used as templates. Membranes were first soaked in dilute aqueous $\mathrm{H}_{2} \mathrm{O}_{2}$ solutions of NIPAM and crosslinker (methylene bisacrylamide) to fill the pores, superficially dried, irradiated with UV, and then soaked in water to remove unreacted monomer and non-crosslinked oligomers. The cylindrical microgels were then released by dissolving the polycarbonate membranes in methylene chloride and the microgel particles were concentrated by filtration and then washed in a series of solvents, after which the microcylinders were stored in water under refrigeration. Optical microscopy confirmed the dimensions of the product microcylinders approximately matched those of the templating pores.

Acknowledgments The author is indebted to Professor Markus Antonietti for his hospitality and support and to the Max Planck Society for their support during a sabbatical leave at the Max Planck Institute of Colloids and Interfaces. The author also gratefully acknowledges the support of Eastern Michigan University and AFOSR Grant No. FA9550-08-1-0431 for support during this sabbatical leave.

\section{References}

1. Zholobenko VL, Khodakov AY, Imperor-Clerc M, Durand D, Grillo I (2008) Initial stages of SBA-15 synthesis: an overview. Adv Colloid Interface Sci 142:67-74

2. Vaudry F, Di Renzo F, Espiau P, Fajula F, Schulz Ph (1997) Aluminum-rich zeolite beta. Zeolites 19:253-258

3. Gontier S, Tuel A (1995) Synthesis and characterization of Ti-containing mesoporous silicas. Zeolites 15:601-610

4. Murphy AR, Fréchet JMJ (2007) Organic semiconducting oligomers for use in thin film transistors. Chem Rev 107:10661096

5. Stein A (2001) Sphere templating methods for periodic porous solids. Micropor Mesoporous Matls 44-45:227-239

6. Holland BT, Blanford CF, Do T, Stein A (1999) Synthesis of highly ordered, three-dimensional, macroporous structures of amorphous or crystalline inorganic oxides, phosphates, and hybrid composites. Chem Mater 11:795-805

7. Velev OD, Kaler EW (2000) Structured porous materials via colloidal crystal templating: from inorganic oxides tometals. Adv Mater 12:531-534

8. John VJ, Simmons B, McPherson GL, Bose A (2002) Recent developments in materials synthesis in surfactant systems. Curr Opin Colloid Interface Sci 7:288-295

9. Henze HP, Kaler EW (2003) Polymerization of and within selforganized media. Curr Opin Colloid Interface Sci 8:164-178

10. Yan F, Texter J (2006) Polymerization of and in mesophases. Adv Colloid Interface Sci 128-130:27-35
11. Tirrell M, Kokkoli E, Biesalski M (2002) The role of surface science in bioengineered materials. Surf Sci 500:61-83

12. Debord JD, Lyon LA (2000) Thermoresponsive photonic crystals. J Phys Chem B 104:6327-6331

13. Takeoka Y, Watanabe M (2002) Polymer gels that memorize structures of mesoscopically sized templates. Dynamic and optical nature of periodic ordered mesoporous chemical gels. Langmuir 18:5977-5980

14. Barry RA, Wiltzius P (2006) Humidity-sensing inverse opal hydrogels. Langmuir 22:1369-1374

15. Lee YJ, Braun PV (2003) Tunable inverse opal hydrogel $\mathrm{pH}$ sensors. Adv Mater 15:563-566

16. Lee YJ, Pruzinsky SA, Braun PV (2004) Glucose-sensitive inverse opal hydrogels: analysis of optical diffraction response. Langmuir 20:3096-3106

17. Matsubara K, Watanabe M, Takeoka Y (2007) A thermally adjustable multicolor photochromic hydrogel. Angew Chem Int Ed 46:1688-1692

18. Sakai T, Takeoka Y, Seki T, Yoshida R (2007) Organized monolayer of thermosensitive microgel beads prepared by double-template polymerization. Langmuir 23:8651-8654

19. Tsuji S, Kawaguchi H (2005) Colored thin films prepared from hydrogel microspheres. Langmuir 21:8439-8442

20. Texter J (2003) Polymer colloids in photonic materials. Comptes Rend Chimie 6:1425-1433

21. Hiltner PA, Krieger IM (1969) Diffraction of light by ordered suspensions. J Phys Chem 73:2386-2389

22. Vanderhoff JW, van den Hull HJ, Tausk RJM, Overbeek JThG (1970) In: Goldfinger G (ed) Clean surfaces: their preparation and characterization for interfacial studies. Dekker, New York, $p 15$

23. Xu X, Friedman G, Humfeld KD, Majetich SA, Asher SA (2002) Polymerized crystalline colloidal array chemical-sensing materials for detection of lead in body fluids. Chem Mater 14:1249-1256

24. Xu XL, Asher SA (2004) Mesoscopic monodisperse ferromagnetic colloids enable magnetically controlled photonic crystals. J Am Chem Soc 124:13864-13868

25. Foulger SH, Katha S, Sweryda-Krawiec B, Baughman TW, Ballato JM, Jiang P, Smith DW Jr (2000) Robust polymer colloidal crystal photonic bandgap structures. Optics Lett 25:1300-1302 doi:10.1364/OL.25.001300

26. Foulger SH, Jiang P, Lattam AC, Smith DW Jr, Ballato J (2001) Mechanochromic reponse of ply(ethylene glycol) mthacrylate hdrogel ecapsulated cystalline clloidal arays. Langmuir 17:60236026

27. Evanoff DD Jr, Hayes SE, Ying YR, Shim GH, Lawrence JR, Carroll JB, Roeder RD, Houchins JM, Huebner CF, Foulger SH (2007) Functionalization of crystalline colloidal arrays through click chemistry. Adv Mat 19:3507-3509

28. Pan GS, Kesavamoorthy R, Asher SA (1997) Optically nonlinear Bragg diffracting nanosecond optical switches. Phys Rev Lett 78:3860-3863

29. Weissman JW, Sunkara HB, Tse AS, Asher SA (1996) Thermally switchable periodicities and diffraction from mesoscopically ordered materials. Science 274:959

30. Pan GS, Kesavamoorthy R, Asher SA (1998) Nanosecond switchable polymerized crystalline colloidal array Bragg diffracting materials. J Am Chem Soc 120:6525-6530

31. Asher SA (1985) U.S. Patent 5,452,123

32. Lee K, Asher SA (2000) Photonic crystal chemical sensors: $\mathrm{pH}$ and ionic strength. J Am Chem Soc 122:9534-9537

33. Holtz JH, Asher SA (1997) Polymerized colloidal crystal hydrogel films as intelligent chemical sensing materials. Nature 389:829 832

34. Holtz JH, Holtz JSW, Munro CH, Asher SA (1998) Intelligent polymerized crystalline colloidal arrays: novel chemical sensor materials. Anal Chem 70:780-791 
35. Foulger SH, Jiang P, Smith DW Jr, Ballato J (2001) Photonic band-gap composites. Adv Mat 13:1898-1901

36. Stephen H. Foulger, Amanda C. Lattam, Ping Jiang, and John Ballato, David E. Dausch, Sonia Grego, and Brian R. Stoner, SPIE 2002

37. Foulger SH, Jiang P, Lattam AC, Smith DW, Ballato J, Dausch DE, Grego S, Stoner BR (2003) Photonic crystal composites with reversible high-frequency stop band shifts. Adv Mat 15:686-689

38. Lawrence JR, Shim GH, Jiang P, Han MG, Ying Y, Foulger SH (2005) Photonic crystal composites with reversible high-frequency stop band shifts. Adv Mat 17:2344-2349

39. Norton J, Han MG, Jiang P, Creager S, Foulger SH (2007) Electrochemical tuning the optical properties of crystalline colloidal arrays composed of poly(3,4-ethylenedioxythiophene) coated silica particles. J Mat Chem 17:1149-1153

40. Ying YR, Xia JQ, Foulger SH (2007) Pressure tuning the optical transmission properties of photonic band gap composites. Appl Phys Lett 90:071110

41. Rodríguez-Abreu C, Lazzari M (2008) Emulsions with structured continuous phases. Curr Opin Colloid Interface Sci 13:198-205

42. Bing Z, Lee JY, Choi SW, Kim JH (2007) Preparation of porous $\mathrm{CaCO} 3 / \mathrm{PAM}$ composites by $\mathrm{CO} 2$ in water emulsion templating method. Eur Polym J 43:4814-4820

43. Noble PF, Cayre OJ, Alargova RG, Velev OD, Paunov VN (2004) Fabrication of "hairy" colloidosomes with shells of polymeric microrods. J Am Chem Soc 126:8092-8093

44. Cayre OJ, Noble PF, Paunov VN (2004) Fabrication of novel colloidosome microcapsules with gelled aqueous cores. J Mater Chem 14:3351-3355

45. Duan HW, Wang DY, Sobal NS, Giersig M, Kurth DG, Möhwald H (2005) Magnetic colloidosomes derived from nanoparticle interfacial self-assembly. Nano Lett 5:949-952

46. Dinsmore AD, Hsu MF, Nikolaides MG, Marquez M, Bausch AR, Weitz DA (2002) Colloidosomes: selectively permeable capsules composed of colloidal particles. Science 298:1006-1009

47. Liu HX, Wang CY, Gao QX, Liu XX, Tong Z (2008) Fabrication of novel core-shell hybrid alginate hydrogel beads. Int $\mathrm{J}$ Pharmaceutics 351:04-112
48. Zhang FJ, Cheng GX, Ying XG (2006) Emulsion and macromolecules templated alginate based polymer microspheres. Reactive Func Polym 66:712-719

49. Rill RL, Locke BR, Liu Y, Dharia J, Van Winkle D (1996) Protein electrophoresis in polyacrylamide gels with templated pores. Electrophoresis 17:1304-1312

50. Anderson D M, Strom P (1989) In El-Nokaly MA, Ed Polymer Association Structures: Microemulsions and Liquid Crystals, American Symposium Series 384:204-224 American Chemical Society:Washington, DC

51. Rill RL, Van Winkle DH, Locke BR (1998) Templated pores in hydrogels for improved size selectivity in gel permeation chromatography. Anal Chem 70:2433-2438

52. Clapper JD, Guymon CA (2007) Physical behavior of crosslinked PEG hydrogels photopolymerized within nanostructured lyotropic liquid crystalline templates. Macromolecules 40:11011107

53. Lester CL, Smith SM, Colson CD, Guyman CA (2003) Physical properties of hydrogels synthesized from lyotropic liquid crystalline templates. Chem Mater 15:3376-3384

54. De Pierro MA, Carpenter KG, Guymon CA (2006) Influence of polymerization conditions on nanostructure and properties of polyacrylamide hydrogels templated from lyotropic liquid crystals. Chem Mater 18:5609-5617

55. Malmsten M, Lindman B (1992) Self-assembly in aqueous block copolymer solutions. Macromolecules 25:5440-5445

56. Texter J, Schwarz R (2005) Fouling release and antifouling coatings derived from thermoreversible gels. Polym Preprints 46:1238-1239

57. Singh N, Lyon LA (2007) Au nanoparticle templated synthesis of pNIPAm nanogels. Chem Mater 19:719-726

58. Hong JS, Vreeland WN, DePaoli Lacerda DS, Locascio LE, Gaitan M, Raghavan SR (2008) Liposome-templated supramolecular assembly of responsive alginate nanogels. Langmuir 24:4092-4096

59. Yan H, Nishino M, Tsuboi Y, Kitamura N, Tsujii K (2005) Template-guided synthesis and individual characterization of poly (N-isopropylacrylamide)-based microgels. Langmuir 21:7076-7079 